\title{
In-situ small-angle X-ray scattering study of the Perovskite- type carbide precipitation behavior in a carbon-containing intermetallic TiAl alloy using synchrotron radiation
}

\author{
E. Schwaighofer ${ }^{1}$, P. Staron ${ }^{2}$, B. Rashkova ${ }^{1}$, A. Stark ${ }^{2}$, N. Schell ${ }^{2}$, H. Clemens ${ }^{1}$, and S. Mayer ${ }^{1}$ \\ ${ }^{1}$ Department of Physical Metallurgy and Materials Testing, Montanuniversität Leoben, 8700 Leoben, Austria \\ 2Institute of Materials Research, Helmholtz-Zentrum Geesthacht, 21502 Geesthacht, Germany
}

Intermetallic $\gamma$-TiAl based alloys of the latest generation, e.g. TNM alloys with a nominal composition of $\mathrm{Ti}-43.5 \mathrm{Al}-4 \mathrm{Nb}-1 \mathrm{Mo}-0.1 \mathrm{~B}$ (in at.\%), exhibit the potential to be used in modern high-performance combustion engines due to their low density, high strength and creep-resistance as well as their good oxidation properties at elevated temperatures. Alloying with $\mathrm{C}$ can further improve the high-temperature performance both by solid solution hardening and/or carbide formation. In this study, starting from a supersaturated TNM-1C alloy the precipitation behavior and thermal stability of Perovskite-type carbides $\mathrm{Ti}_{3} \mathrm{AlC}$ during isothermal annealing at $750{ }^{\circ} \mathrm{C}$ and ensuing re-heating to $1200{ }^{\circ} \mathrm{C}$ is quantified by means of an in-situ small-angle X-ray scattering (SAXS) experiment using synchrotron radiation. Complementary, the formed hierarchical structures on the nano-scale, i.e. p-type carbide precipitates within ultra-fine $\gamma$-lamellae of the $\alpha_{2} / \gamma$-colonies, were investigated by means of monochromatic high-energy X-ray diffraction (HEXRD) in combination with transmission electron microscopy (TEM). Additionally, an explanation of an obtained diffraction phenomenon is given, i.e. streak formation which is caused by the very small lamellar spacing of the $\gamma$-phase within the $\alpha_{2} / \gamma$-colonies. It was also found that the geometrically well-defined nanostructure allows a correlation between the $\gamma$-laths thickness and a characteristic dimension of p-type carbides.

The experiments were carried out at the HZG high-energy materials science beamline HEMS (P07). A sketch of the experimental setup is depicted in Fig. 1. The monochromatic beam with a mean energy of $100 \mathrm{keV}$ is guided through the sample which is situated in a modified quenching and deformation dilatometer device DIL 805 A/D from Bähr Thermoanalyse, Germany. The diffraction patterns are recorded with a Perkin Elmer XRD 1622 flat panel detector in experimental hutch EH 3, whereas a mar345 Image Plate detector located behind in EH 4 is used to record the signal from small-angle scattering. The cylindrical sample with a size of $5 \mathrm{~mm}$ in diameter and $10 \mathrm{~mm}$ in length was heated inductively. The SAXS data were recorded with a sample-to-detector distance of $11.654 \mathrm{~m}$ and a frame rate of 20 images per hour. Slits made of pure tungsten minimized undesired scattering and, thus, ensured a low background. Data evaluation was performed by means of the program Fit2D with respect to azimuthal integration $[1,2]$ followed by a fitting procedure of the derived scattering curves with the program SANSFit based on a least-square method iteration as conducted in Ref. [3]. More details about the experimental conditions and the obtained results are given in the original full-length paper in [4].

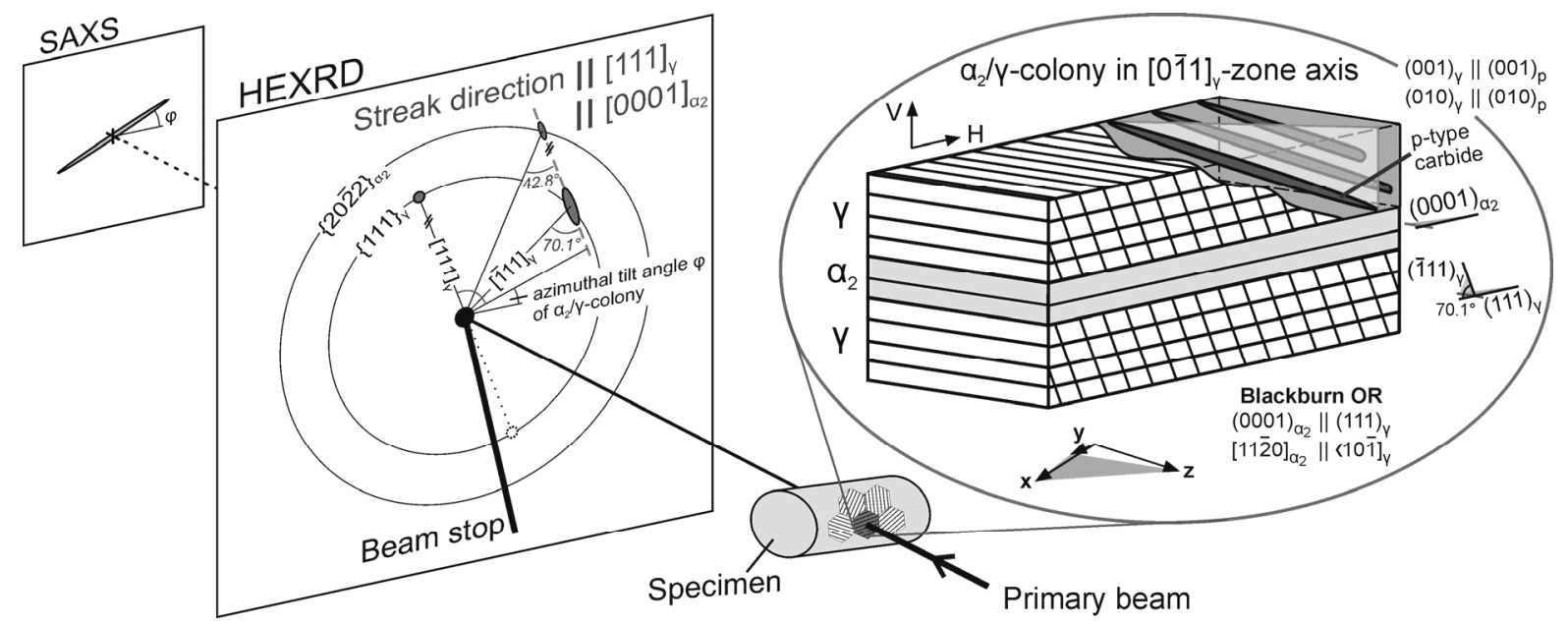

Figure 1: Experimental setup of the conducted synchrotron experiment. $\mathrm{H}=$ horizontal, $\mathrm{V}=$ vertical (related to crystal-coordinate system of the $\gamma$-phase). For explanations see text. 
The conducted in-situ SAXS experiment starting from a supersaturated TNM-1C alloy allows to determine the precipitation behavior during isothermal annealing at $750{ }^{\circ} \mathrm{C}$ as well as the thermal stability of p-type carbides during further heating to $120{ }^{\circ} \mathrm{C}$ with a rate of $2 \mathrm{~K} / \mathrm{min}$ (Fig. 2a). It was found that p-type carbide formation takes place after an incubation time of 30 min within the $\gamma$-laths previously formed from supersaturated $\alpha_{2}$-grains. Thus, the phase-transformation sequence takes the form $\alpha_{2, \mathrm{ss}} \rightarrow \alpha_{2}+\gamma$ and $\gamma \rightarrow \mathrm{p}-\mathrm{Ti}_{3} \mathrm{AlC}$. After ageing for $4 \mathrm{~h}$ at $750{ }^{\circ} \mathrm{C}$, the carbide precipitates exhibit a mean radius of $1.7 \mathrm{~nm}$ - obeying a monodisperse size distribution - which correlates with a carbide length of $23.8 \mathrm{~nm}$ and a mean $\gamma$-lamellae thickness of $13.8 \mathrm{~nm}$ (see also TEM-results in Figs. 2b,c). Thereby, the thickness $D$ of $\gamma$-laths and the radius $R$ of p-type carbides can be correlated by the geometrical relation $D=2 \mu R \times \cos \left(54.7^{\circ}\right)$ according to the orientation relationship $(001)_{\gamma} \|(001)_{\mathrm{p}}$ and $(010)_{\gamma} \|(010)_{\mathrm{p}}$. The needle-shaped p-type carbides exhibit a typical size aspect ratio of $\mu=7$ as verified by TEM (Fig. 2c). The geometrical correlation is valid as long as the size of p-type carbides is controlled by the small dimension of $\gamma$-lamellae. Complementary, the data from SAXS are in good agreement with the evaluation of diffraction streaks from the twodimensional HEXRD patterns using the Scherrer Equation (Fig. 2d). There, the occurrence of diffraction streaks, i.e. anisotropic peak broadening, was found to originate from the finite thickness of $\gamma$-laths and the geometrical relation between the monochromatic X-ray beam and the structure of an $\alpha_{2} / \gamma$-colony within the polycrystalline TNM-1C material. Diffraction streaking implies that the $70.1^{\circ}$ tilted $(-111) \gamma$ lattice planes are in diffraction condition, which do not exhibit a strong superordinate structure when compared to (111) $\gamma$ lattice planes which are by definition parallel to $(0001) \alpha_{2}$.
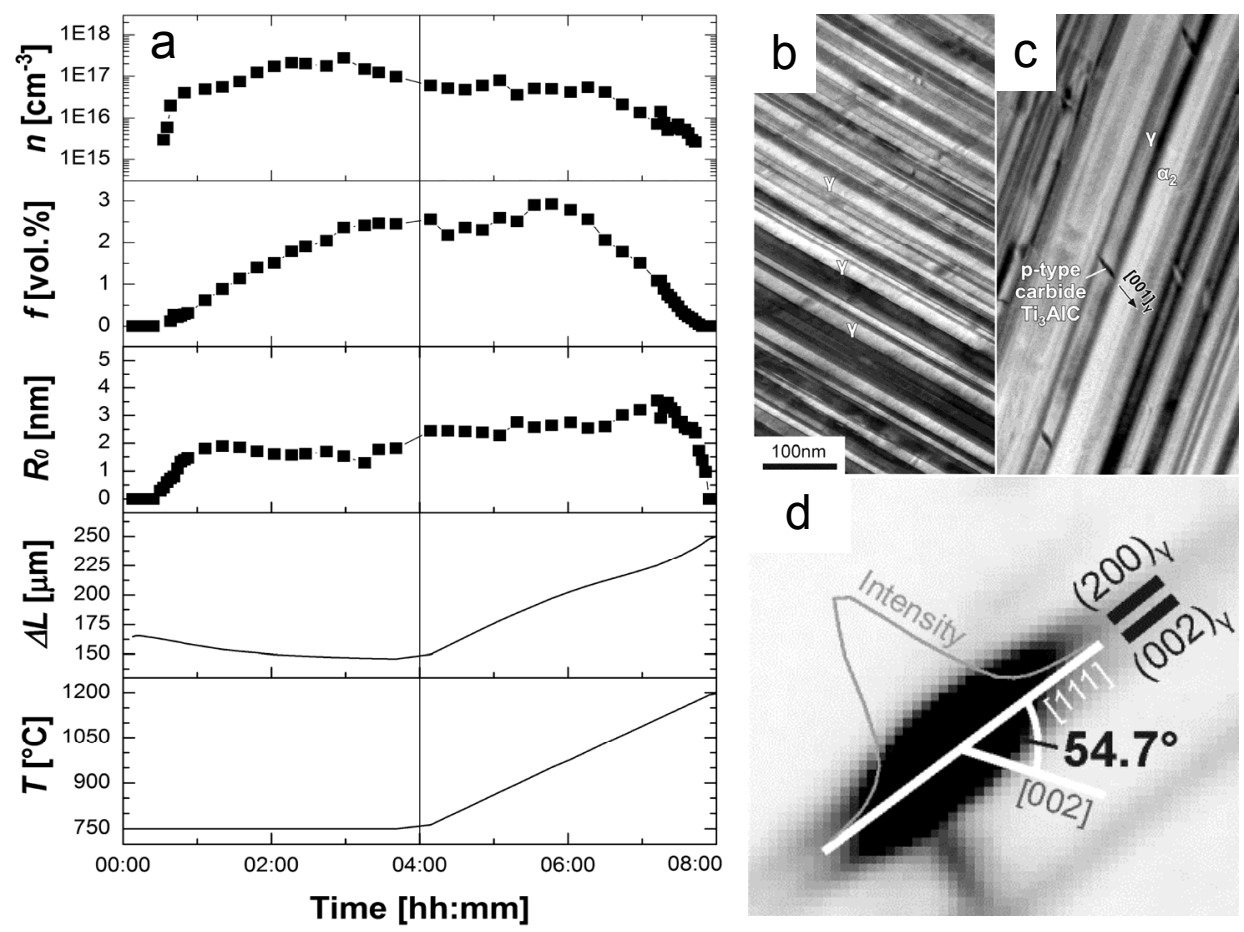

Figure 2: a) Progress of temperature $T$ and length change $\Delta L$ of the dilatometer sample during the heattreatment. Additionally, the median radius $R_{0}$ of the needle-shaped p-type carbide precipitates, their volume fraction $f$ in relation to the $\gamma$-phase content and their number density $n$ are given. b) TEM bright-

field (BF) image of an $\alpha_{2} / \gamma$-colony in [11-20 $]_{\alpha 2}$ and $\left.<10-1\right]_{\gamma}$ zone axis. c) TEM BF image of p-type carbides aligned within a characteristic $\gamma$-lath. d) The HEXRD image section after precipitation annealing at $750{ }^{\circ} \mathrm{C}$ for $4 \mathrm{~h}$ corresponds to a detail of the streaked $(002)_{\gamma}$ and $(200)_{\gamma}$ reflections.

\section{References}

[1] A. P. Hammersley, S. O. Svensson, M. Hanfland, A. N. Fitch, and D. Häusermann, High Pressure Res. 14, 235 (1996).

[2] T. Schmoelzer, K.-D. Liss, P. Staron, S. Mayer, and H. Clemens, Adv. Eng. Mat. 13, 685 (2011).

[3] P. Staron, U. Christoph, F. Appel, and H. Clemens, Appl. Phys. A 74, 1163 (2002).

[4] E. Schwaighofer, P. Staron, B. Rashkova, A. Stark, N. Schell, H. Clemens, and S. Mayer, submitted to Acta Mater. (2014). 